\title{
Resolución de problemas, habilidades y rendimiento académico en la enseñanza de la matemática
}

Gamarra Astuhuaman, Guillermo; Pujay Cristóbal, Oscar Eugenio

Resolución de problemas, habilidades y rendimiento académico en la enseñanza de la matemática

Revista Educación, vol. 45, núm. 1, 2021

Universidad de Costa Rica, Costa Rica

Disponible en: http://www.redalyc.org/articulo.oa?id=44064134019

DOI: https://doi.org/10.15517/revedu.v45i1.41237

Esta obra está bajo una Licencia Creative Commons Atribución-NoComercial-SinDerivar 3.0 Internacional. 


\title{
Resolución de problemas, habilidades y rendimiento académico en la enseñanza de la
} matemática

\author{
Problem Solving, Skills and Academic Performance when Teaching Mathematics
}

Guillermo Gamarra Astubuaman

Universidad Nacional Daniel Alcides Carrión, Perú

ggamarraastuhuaman@gmail.com

(D) http://orcid.org/0000-0002-5385-8655

Oscar Eugenio Pujay Cristóbal

Universidad Nacional Daniel Alcides Carrión, Perú

oscareugeniopujaycristobal@gmail.com

(iD) http://orcid.org/0000-0002-2051-9403
DOI: https://doi.org/10.15517/revedu.v45i1.41237

Redalyc: http://www.redalyc.org/articulo.oa?id=44064134019

Recepción: 14 Abril 2020

Aprobación: 23 Septiembre 2020

\section{Resumen:}

En la presente investigación se muestra los resultados de un estudio aplicado a 115 estudiantes de universidad en la asignatura de Lógico Matemática II. El objetivo de la investigación es determinar si el método de resolución de problemas influye en el desarrollo de habilidades cognitivas y el mejoramiento del rendimiento académico de un grupo de estudiantes de educación superior. Se seleccionaron dos grupos, uno denominado grupo experimental (GE) y el otro grupo control (GC). A ambos grupos se les aplicó dos pruebas de conocimiento, denominadas pre-test y post-test. Después de ser aplicado el pre-test, se implementó el método de resolución de problemas al grupo experimental, cuya duración fue de 16 semanas; una vez finalizado el desarrollo de todo el contenido programático se procedió a aplicar el post-test. Los datos recopilados fueron el resultado de las calificaciones obtenidas de las evaluaciones; estas fueron valoradas de manera cualitativa y cuantitativa, y analizadas haciendo uso de procedimientos estadísticos. La media de las calificaciones obtenidas por ambos grupos en el pre-test y post-test fueron 11.25 (GE) y 11.64 (GC), y 15.08 (GE) y $11.42(\mathrm{GC})$, respectivamente; en cuanto al aspecto cualitativo, el grupo experimental mejoró en las habilidades de cálculo, identificación de variables, diseño de estrategias, deducciones e inferencias, aunque la mejora fue poco significativa. Del análisis inferencial se obtuvo un coeficiente de Pearson igual a 0.771 y un p-valor de 0.000 , lo que evidencia la influencia del método aplicado en las variables desarrollo de las habilidades cognitivas y el mejoramiento del rendimiento académico.

Palabras clave: Método, Matemática, Habilidad cognitiva, Enseñanza, Aprendizaje.

\section{Abstract:}

This article shares the results of a study among 115 university students enrolled in a Mathematical Logic II course. The objective of this study was to determine if problem-solving methods influence cognitive skills development and help improve academic performance in a group of university students. The students were divided into two groups- the Experimental Group (EG) and the Control Group (CG) and were administered two knowledge tests - a pre-test and post-test. After the Experimental Group took the pre-test, they were taught the problem-solving method during 16 weeks. Upon completion of the course content, the students took a post-test. The collected data collected includes the grades from these assessments which were evaluated both qualitatively and quantitatively and analyzed through descriptive and inferential statistics. The pre-test and post-test mean scores for both groups was 11.25 (GE) and 11.64 (GC); and 15.08 (GE) and 11.42 (GC), respectively. Regarding the qualitative aspect, although the experimental group improved its calculation, variable identification, strategy design, deduction and inference skills, it did not reveal a significant improvement. An inferential analysis reveals a Pearson's coefficient equal to 0.771 and with a p-value of 0.000 , thus proving the impact of using the applied method on cognitive skills development and improved academic performance.

KEYworDs: Method, Mathematics, Cognitive Ability, Teaching, Learning. 


\section{INTRODUCCIÓN}

La matemática constituye un área del saber humano de gran importancia e interés, no solo por sus aportaciones al desarrollo tecnológico y científico, sino también porque son indispensables en la formación académica de todo individuo, pues muchos de los futuros profesionales requieren ineludiblemente de los conceptos matemáticos para poder desenvolverse en el ámbito laboral (Pino, 2013). A pesar de que las matemáticas se encuentran presentes en la cotidianidad, existe un notable desinterés por parte de muchas personas a estudiar carreras que estén vinculadas a estas, en consecuencia, el interés en la formación profesional se centra hacia otras áreas, algunas veces genera un déficit de profesionales en el mercado laboral; por lo tanto, el reto de las instituciones educativas, sobre todo en las primeras etapas de formación académica, es lograr constantemente que el estudiantado sienta el deseo de interesarse por el aprendizaje de las matemáticas (Carrasco y Sánchez, 2016). En este orden de ideas, el presente artículo tiene como finalidad indagar sobre algunos aspectos teóricos sobre la resolución de problemas en el proceso de enseñanza y aprendizaje de las matemáticas o la lógica matemática, y de brindar, de alguna forma, una visión crítica que constituya un aporte al mejoramiento en la enseñanza (Serradó, Cardeñoso y Azcárate, 2005).

Por otra parte, Echemendia, Ramos y Delgado (2017) desarrollaron una investigación sobre la resolución de problemas en la enseñanza de la biología, y establecieron que dicha metodología está orientada a enseñar a pensar constituyendo una herramienta que contribuye a la formación de personas en un nuevo tipo de sociedad; también, resaltaron que es significativo el número de investigaciones relacionadas con la resolución de problemas en las áreas de matemática y física. La resolución de problemas es uno de los aspectos en los que el estudiantado presenta mayor dificultad para consolidar los conocimientos requeridos en matemáticas, debido al mecanicismo en el que casi siempre se desarrolla el proceso de enseñanza; tal proceso muchas veces no es orientado a aplicaciones de la vida cotidiana, sobre todo en las primeras etapas de formación académica. A su vez, Calvo (2008), señala que la formación del estudiantado ha de ser desarrollada bajo un ambiente favorable que le permita siempre estar interesado en el aprendizaje de la matemática, haciendo uso de solo abstracciones que motiven a consolidar los conceptos fundamentales. Por otra parte, existen docentes que, de acuerdo con su experiencia de formación, continúan usando métodos de enseñanza tradicionales, lo cual hace que la enseñanza de conceptos sea vista por sus estudiantes como algo que carece de sentido y poco útil.

En el trabajo de Pifarré y Sanuy (2001), se afirma que los buenos resolutores de problemas matemáticos poseen estrategias orientadas a acciones que les permiten solventar las dificultades encontradas durante el proceso de resolución; sin embargo, cada problema presenta diferentes niveles de dificultad, por lo que el resolutor emplea, como recurso, acciones y procesos análogos, y a partir de estos genera nuevos modelos que facilitan el hallazgo de soluciones que son lógicas y contrastables. Estas soluciones son estructuradas en fases que hacen más fácil el proceso de enseñanza y aprendizaje; el mismo autor señala que deben diagnosticarse los conocimientos y habilidades previas que posee el estudiantado, y sus capacidades adaptativas a los modelos de resolución.

Para Villalobos (2008), los problemas matemáticos deben poseer no solo procesos algorítmicos, sino también dificultades intelectuales y habilidades cognitivas, con características motivadoras y que estén en consonancia con el contexto real y social del aprendiz. Además, un problema matemático debería ser resuelto o abordado de distintas maneras o resuelto con diferente método, según sea el caso. De igual manera, el autor establece que la resolución de problemas se configura como un aspecto primordial en la enseñanza y el aprendizaje de la educación matemática. Sin embargo, la enseñanza, por medio de resolución de problemas, brinda dos enfoques. El primero identificado por su carácter disciplinar, en el cual los procedimientos y los resultados son precisos, pero se manejan conceptos y procesos que a veces no tienen algún significado tanto para el estudiantado como para el profesorado. El segundo enfoque se centra en los aspectos contextuales dirigidos a la construcción social del aprendizaje, relacionado con el desarrollo de las capacidades y habilidades del alumnado, colocándole por encima de los conceptos y procedimientos. Por otra parte, Kohler (2013) 
señala en su investigación que son clave las estrategias de aprendizaje que el estudiantado emplea en su proceso de formación, puesto que les permite hacer toma de decisiones conscientes y coherentes, que llevan a implementar un conjunto ordenado de operaciones mentales a fin de procesar bien la información para adquirir el aprendizaje y las capacidades para enfrentar situaciones en diferentes contextos.

En una investigación dirigida por Martín, Martínez, Fernández y Bravo (2016), se establece que la capacidad de resolución de problemas facilita a las niñas y a los niños a explorar una diversidad de conceptos matemáticos y formarlos para que puedan describir sucesos cotidianos mediante el pensamiento lógico. Siguiendo las ideas planteadas por dicho autor, la combinación de los juegos y tareas son útiles para que el estudiantado aborde algunos conceptos matemáticos, y puedan identificarse con procesos matemáticos de manera continua, evidenciando un notable interés en cumplir sus actividades. Lo anterior puede ser trasladado al ámbito universitario y hacer del proceso de enseñanza y aprendizaje algo productivo y confortante, cubriendo así las expectativas académicas del estudiantado. El método de resolución de problemas ha de ser enseñado de forma tal que este domine las técnicas y desarrolle sus capacidades, las cuales puedan ser mostradas en su entorno sociocultural y profesional.

El desafío que toda institución educativa enfrenta constantemente es el mejoramiento del rendimiento académico del estudiantado, el cual depende de la manera en que se van dando los resultados de cada aprendizaje y del constante proceso de verificación por medio de la evaluación cualitativa y cuantitativa, (Estrada, 2018).

Por otra parte, a nivel especifico, se tienen estudios como los Chico, Gamboa y Henríquez (2017), en el cual se plantea que el sistema de ejercicios y problemas trigonométricos se caracteriza por ser flexible y adaptable a las condiciones cambiantes del grupo del estudiantado, de forma tal que pueda adaptarse a las necesidades del grupo en general y al alumnado en lo particular. De tal forma, se logra comprobar el desarrollo de habilidades a través de la resolución de problemas en del estudiantado.

Así mismo, Sabonete, Gamboa y Mestre (2016) ofrecen una propuesta didáctica para el diseño de los problemas de matemática, la cual contribuyó a mejorar el rendimiento del alumnado y a emprender exitosamente nuevas situaciones de aprendizaje en esta asignatura a través de la resolución de problemas.

Sin embargo, Roque (2009) desarrolló una investigación en la cual demuestra que existen muchas dificultades en la enseñanza y el rendimiento académico del estudiantado. Sin considerar que, a través de la resolución de problemas, este fortalece y amplía su cultura matemática. Un aspecto fundamental para afrontar diversas situaciones en una sociedad matematizada es lograr ser un/a profesional competente y mejorar la calidad de vida de la sociedad. En esa misma línea de investigación, Sepúlveda, Medina y Sepúlveda (2009), en su estudio determinan que existe un bajo rendimiento académico en la asignatura de matemáticas.

Por ello, el objetivo de la presente investigación se centró en determinar si existe relación entre las habilidades y el rendimiento adquirido por un grupo de estudiantes de universidad a través del método de resolución de problemas y hacer algunas comparaciones con otros estudios reportados.

\section{Procedimientos metodológicos}

El tipo de investigación del presente estudio fue de carácter descriptivo y explicativo, puesto que su principal objetivo consistió en medir el rendimiento académico de una población de estudiantes de la Facultad de Educación de la Universidad Nacional Daniel Alcides Carrión [UNDAC], de la provincia de Pasco, mediante una investigación de tipo cuasi-experimental, debido a que se buscó determinar si existe relación entre las habilidades matemáticas adquiridas por el grupo de estudiantes y su rendimiento académico. También es importante mencionar que la investigación también fue aplicada al campo de la educación, pues su fin fue modificar las conductas del grupo de estudiantes (grupo experimental) mediante la aplicación del método de resolución participativo. 
Se determinó una muestra inicial de 220 estudiantes, mediante la fórmula para poblaciones finitas, con un error de muestra de 5\% y una confiabilidad de 95\%. El grupo estudiantil estaba inscrito al inicio de la experiencia en la asignatura Lógico Matemática II de la UNDAC. Posteriormente, se aplicó un factor de corrección, determinándose una muestra final de 115 estudiantes, debido a que no todos pertenecían a las mismas carreras. Se organizó un sorteo para seleccionar al estudiantado que conformaría un grupo experimental de estudiantes y un grupo de control. El grupo experimental estuvo conformado por 57 estudiantes de las especialidades de Matemática y Física; Computación e Informática; Idiomas (inglés y francés); Lengua y Literatura, y Filosofía-Ciencias Sociales. Mientras que el grupo de control estuvo representado 58 estudiantes de las especialidades de Química y Biología; Historia y Geografía; Inicial, y Primaria de las localidades de Cerro de Pasco, Oxapampa y Yanahuanca.

Una vez seleccionados los grupos participantes de la experiencia, se elaboraron dos pruebas, una aplicada antes de iniciar la experiencia pedagógica, y otra después de aplicar dicha experiencia; ambas pruebas fueron aplicadas a los dos grupos para obtener la información sobre el rendimiento académico del estudiantado que conforman las unidades de análisis del presente estudio antes de aplicar el tratamiento. Después de haberse aplicado el pre-test se procedió a aplicar la propuesta para el grupo experimental, la cual tuvo una duración de siete semanas, y en cada semana se daban seis horas de clases, de las cuales dos horas fueron dedicadas a tratar los aspectos teóricos y cuatro horas a la resolución de problemas. Finalizado el desarrollo del contenido programático, se procedió a aplicar el post-test para obtener la información sobre el rendimiento académico del estudiantado que conforma las unidades de análisis del presente estudio después de aplicar el tratamiento.

$\mathrm{El}$ análisis de información responde a los siguientes criterios y rangos: básico de $00 \mathrm{a} 05$ puntos; intermedio de 06 a 11 puntos; superior de 12 a 17 puntos y avanzado de 18 a 20 puntos. Asimismo, se elaboró un análisis estadístico y los datos fueron analizados por medio del programa estadístico SPSS versión 25.

\section{MÉtodo DE RESOLUCión DE PROBLEMAS}

El método de resolución empleado en el presente estudio consistió en un 33,33\% de horas de clases teóricas a un grupo de estudiantes, denominado grupo experimental. En estas clases teóricas se les permitía hacer intervenciones y a cada estudiante se le asignó material didáctico como guías teórico-prácticas, dirección web de aplicaciones matemáticas, entre otros. El otro 66,67\% de las horas se emplearon en la resolución de problemas con la participación de todo el estudiantado de forma coordinada con el profesorado. Cada estudiante pasaba al pizarrón a compartir la resolución de sus problemas y cada docente se encargaba de guiar la labor, así como también de visualizar las actividades que cada uno iba efectuando.

\section{RESULTADOS Y DISCUSIONES}

Se determinó la validez de las pruebas con un coeficiente de confiabilidad de 0.858 , mostrando un grado de consistencia interna alta, para el pre-test y post-test. A continuación, se presentan los resultados obtenidos de las pruebas pre-test y post-test aplicadas al grupo experimental y al grupo control. 


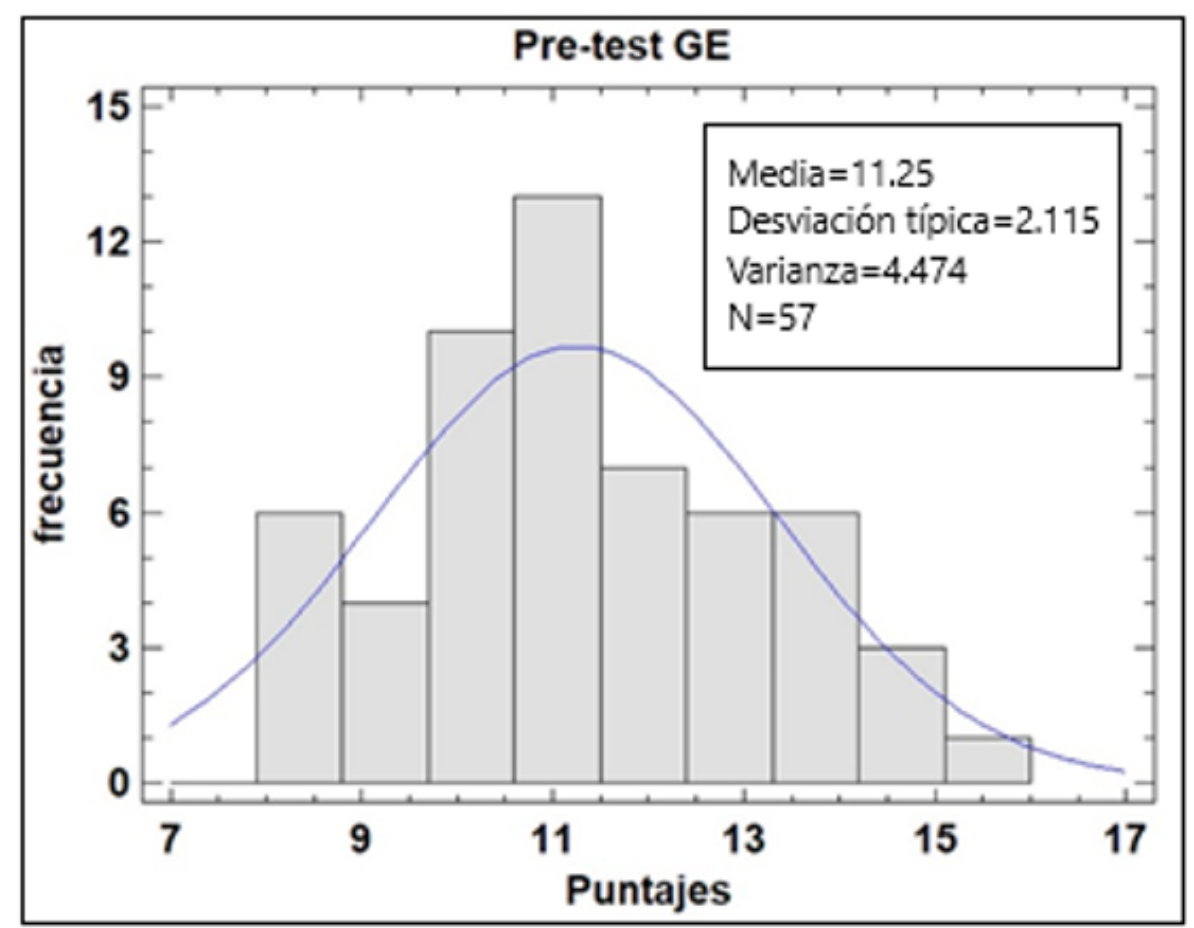

FIGURA 1

Distribución de frecuencias de calificaciones pre-test GE

Fuente: Elaboración propia

En la Figura 1 se muestra la distribución de frecuencias de las calificaciones obtenidas por el grupo experimental. El rango de calificaciones varía desde un valor mínimo de 7 hasta un valor máximo de 16 la media, y la media de dichas calificaciones corresponde a un valor de 11.25, con una desviación estándar de 2.115 y una varianza de 4.474 . El número de estudiantes con buenas calificaciones es relativamente bajo, observándose un mayor número de estudiantes con notas regulares aprobatorias y notas reprobadas, lo cual indica que presentan dificultades a la hora de resolver problemas matemáticos. También se observa, de las evaluaciones, que las estrategias para resolver varios de los problemas por parte del estudiantado, son inadecuadas o no están completamente desarrolladas; posiblemente porque los aspectos conceptuales al momento de presentar el pre-test, no han sido bien comprendidos. 




FIGURA 2

Distribución de frecuencias de calificaciones pre-test GC

Fuente: Elaboración propia

La distribución de las calificaciones obtenidas en la prueba 2 para el grupo de control son mostradas en la Figura 2. Se observa en la gráfica que las calificaciones del grupo de control varían entre un valor mínimo de 6 a un valor máximo igual a 16, con una media de 11.64, por encima del valor obtenido por el grupo experimental. Se obtuvo una desviación estándar de 2.307 con una variancia de 5.323. Con respecto al grupo experimental, el número de estudiantes con calificaciones reprobadas es menor, por lo que el número con calificaciones regulares por encima del promedio es mayor. Se observa un mayor rendimiento académico por parte del grupo de control, aunque el estudiantado de este grupo presenta casi las mismas debilidades conceptuales que estudiantes del grupo experimental, para el pre-test. 


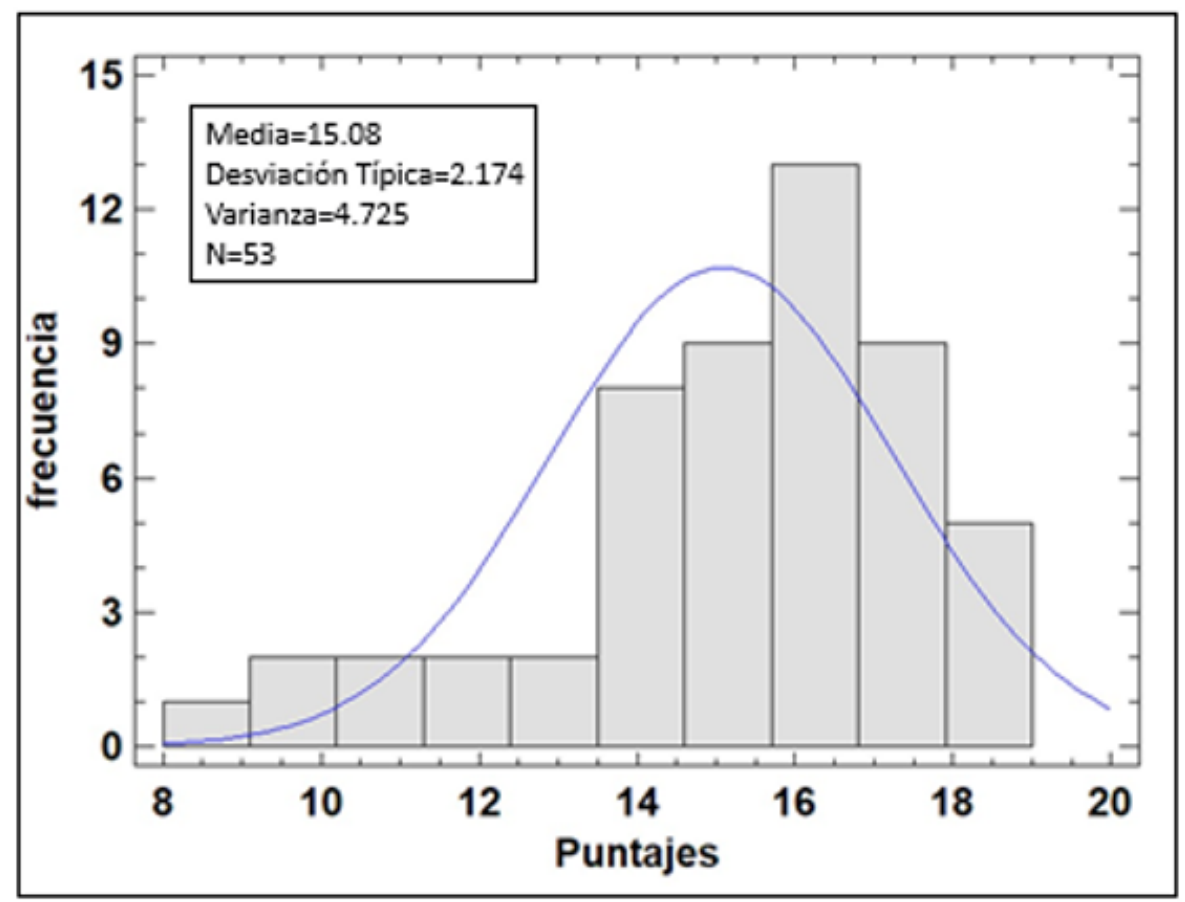

FIGURA 3

Distribución de frecuencias de calificaciones Post-test GE

Fuente: Elaboración propia

En la Figura 3, se detalla la distribución de frecuencia de las calificaciones obtenidas por el grupo experimental, estas varían desde un mínimo de 9 puntos a un máximo de 18 y la media fue de 15.08 , con una desviación estándar de 2.174 y una varianza igual a 4.725. De este resultado se puede observar un aumento considerable en las calificaciones del estudiantado del grupo experimental, con respecto a los resultados obtenidos en el pre-test y los resultados obtenidos por el grupo control. Se observa un aumento del número de estudiantes con calificaciones regulares y calificaciones buenas, en comparación a la primera evaluación. Después de aplicar el método de resolución de problemas y de aplicar el post-test, se observó un mayor dominio por parte del estudiantado del grupo experimental y un aumento de sus habilidades, evidenciado en el desarrollo completo de las soluciones a cada problema planteado y también en las estrategias empleadas al momento de resolver. 


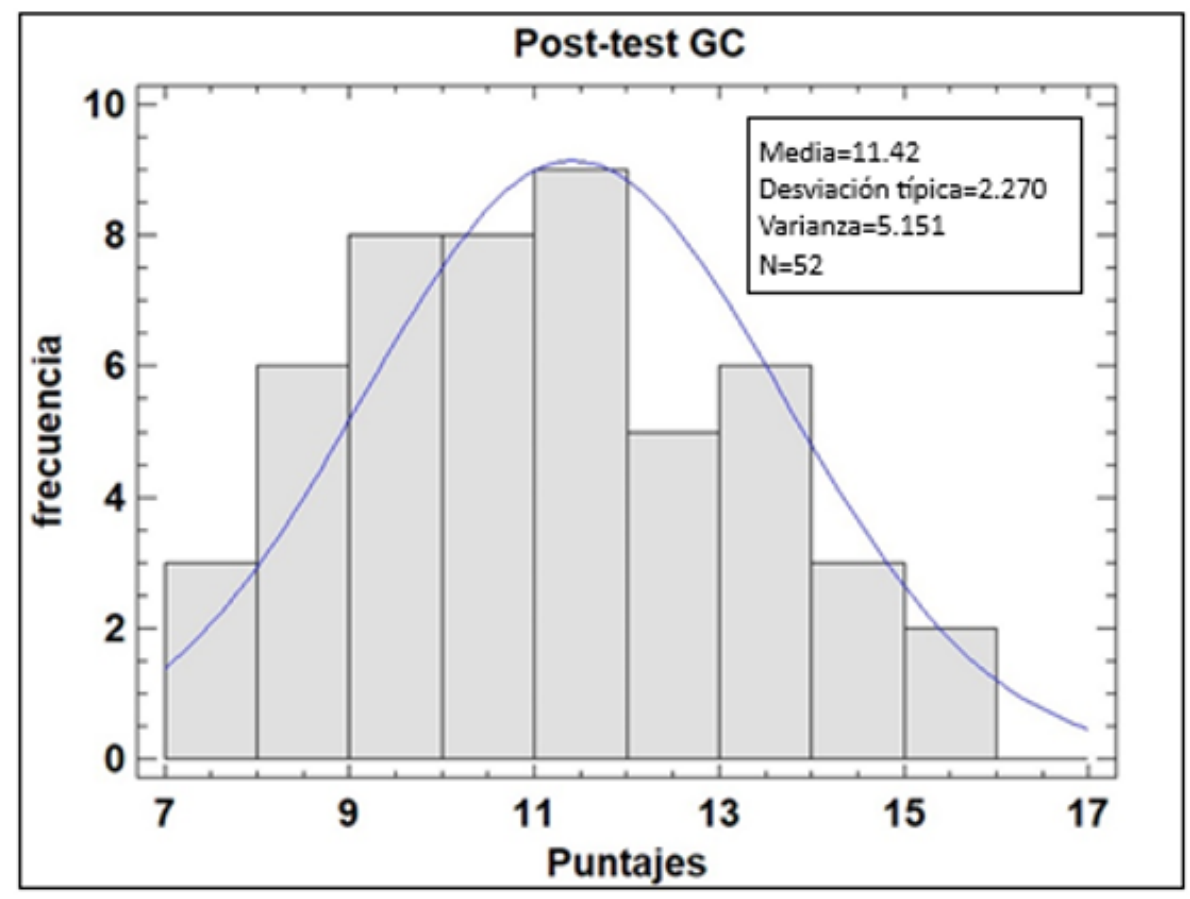

FIGURA 4

Distribución de frecuencias de calificaciones post-test GC

Fuente: Elaboración propia

En la Figura 4 se presenta las distribuciones de las calificaciones del post-test. Las calificaciones para este grupo varían desde el valor mínimo de 7 al valor máximo de 16; en tanto que la media de las calificaciones es de 11.42, por debajo del valor que este grupo obtuvo en el pre-test; con una desviación estándar de 2.270 y una variancia de 5.151. Se observa, con respecto al pre-test aplicado a este grupo, un aumento del número de estudiantes reprobados y una disminución en el promedio de las calificaciones. El estudiantado de este grupo presentó las mismas deficiencias que en el pre-test, incluso experimentó una leve disminución del valor promedio de 11.64 a 11.42. lo que sugiere, que no se empleó ningún cambio en las estrategias de enseñanza y aprendizaje.

\section{Resultado Del Desarrollo de haBilidades}

De las pruebas antes de la aplicación del método de resolución de problemas, se evaluaron algunas de las habilidades desarrolladas por el estudiantado que participó en la experiencia; cada una de estas habilidades representa una dimensión con un determinado número de indicadores, los cuales, a su vez, se califican haciendo uso de una escala de valoración, tal como se muestra en la Figura 5. 


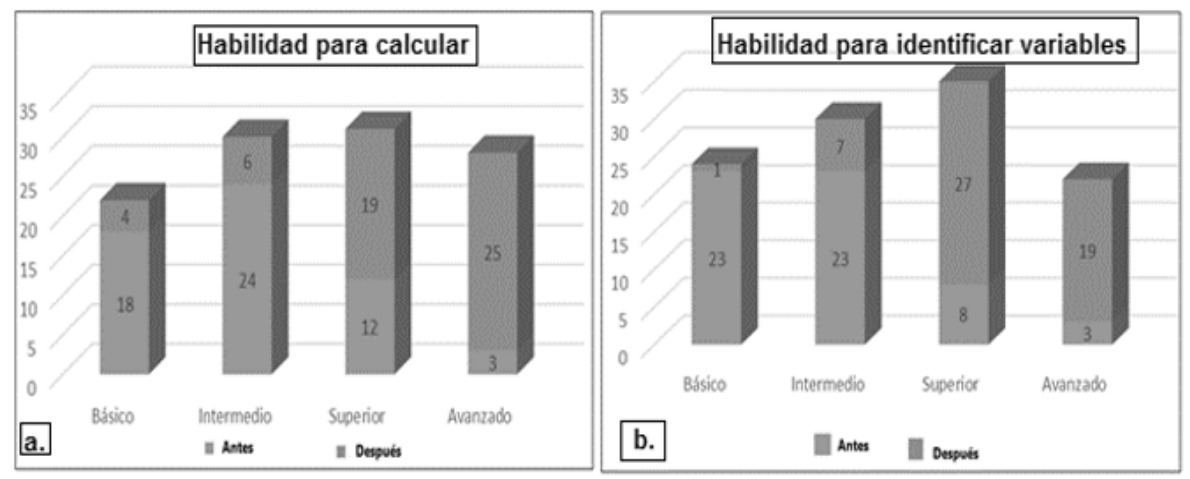

FIGURA 5

Resultados de la evaluación de habilidades, dimensiones cálculo e identificación de variables Fuente: Elaboración propia

En la Figura 5 se muestra los resultados para la dimensión habilidad para calcular, en el pre-test y en el post-test; se observa que una vez aplicado el post-test, aumenta el número de estudiantes con habilidades para desarrollar operaciones relativas al cálculo con mayor facilidad y rapidez. De 12 estudiantes que registraron un nivel superior, la cifra aumentó a 19 estudiantes; en la pre-test solo tres estudiantes obtuvieron la calificación del nivel avanzado, pero en el post-test el número se elevó a 25; observándose un notable avance en el rendimiento del estudiantado.

Con respecto a la dimensión habilidad para identificar variables, en la Figura 5 se observa los resultados del pre-test y el post-test. En el pretest, el número de estudiantes que se le dificultaba identificar los tipos de variables era de 46 estudiantes: 23 con un nivel básico, y 23 con nivel intermedio en la habilidad referida. Es decir, el nivel básico correspondía a aquellos/as estudiantes que identificaban incorrectamente las variables, y el nivel intermedio a los que identificaban las variables explícitas, pero no las implícitas. Después de aplicado el post-test, los resultados se revirtieron, por lo que, de ocho estudiantes en el nivel superior, el número aumentó a 27; y de tres estudiantes en el nivel avanzado, la cifra se incrementó a 19 estudiantes.

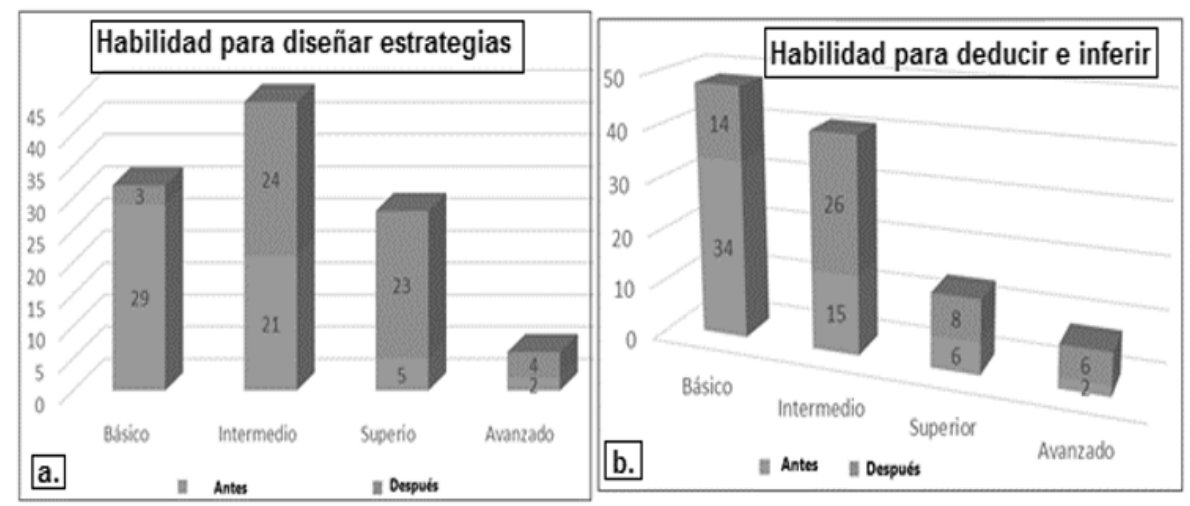

FIGURA 6

Resultados de la evaluación de habilidades, dimensiones diseño de estrategias y deducción e inferencias Fuente: Elaboración propia

Posteriormente, en la Figura 6 se dan los resultados de la evaluación de las dimensiones habilidad para diseñar estrategias y habilidad para deducir e inferir, respectivamente, tanto del pre-test como de post-test. Primeramente, se nota en la Figura 6, que el número de estudiantes con habilidades para diseñar estrategias era bajo al momento del pre-test, con una cantidad de cinco estudiantes que elaboraron estrategias para resolver problemas, pero no adecuadamente, y dos estudiantes, que sí propusieron varias estrategias y lograban soluciones coherentes. En este caso, solo dos estudiantes más adquirieron la habilidad de proponer varias 
estrategias para resolver problemas particulares y 18 estudiantes más, aunque no lograron elaborar estrategias adecuadamente, al menos mostraron el interés por querer aprender al respecto.

Respecto a la sección b de la Figura 6. referido a la habilidad para efectuar inferencias y deducciones, el número de estudiantes que no sustenta las soluciones con argumentos válidos es de 34, representando el nivel básico, pero ya para el nivel intermedio esta cifra aumenta de 15 a 26 estudiantes; sin embargo, el incremento del número de estudiantes de los niveles superior y avanzado va de seis a ocho estudiantes, mientras que, para el nivel avanzado, la cifra de estudiantes se incrementó de dos a seis estudiantes.

Para las dimensiones diseño de estrategias y deducción e inferencias, se muestra que el aumento de estas habilidades en el estudiantado no es tan significativo como en el que se evidencia para las dimensiones habilidades para calcular y habilidades para identificar variables, esto sugiere que en estas dos dimensiones el estudiantado requiere de mayor tiempo para adquirir destrezas y poder resolver los problemas matemáticos con mayor seguridad. También los resultados de esta investigación, en relación con las habilidades cognitivas adquiridas por cada estudiante, concuerdan con trabajos como el de Cerda, Pérez, Romera, Ortega y Casas (2017), donde es reportada la influencia que tiene las habilidades cognitivas en el rendimiento académico, por lo que es necesario implementar métodos de aprendizaje que impacten positivamente en dicho mejoramiento.

De los párrafos anteriores, se hace evidente la mejora que ha tenido el grupo experimental en cuanto al desarrollo de habilidades y aumento en el rendimiento académico, sin embargo, al hacer uso de la estadística inferencial, se ha determinado el coeficiente de correlación de Pearson, el cual varía entre [-1, 1], es decir, $-1 \leq \mathrm{C} \leq 1$; el valor obtenido, mediante el programa estadístico es de 0.771 , representando una correlación alta, y el p-valor obtenido por medio de este tratamiento estadístico es de 0.000 , con un valor de significancia $\alpha=0.05_{2 \text { colas; }}$ por lo tanto, se corrobora una vez más la relación entre las variables desarrollo de habilidades cognitivas y el rendimiento académico una vez aplicado el método de resolución de problemas en el aula de clases. Estos resultados confirman los resultados obtenidos por Sabonete et al. (2016), al determinar el coeficiente de correlación de Pearson de las variables estrategias de enseñanza de las matemáticas y el rendimiento académico del estudiantado, el cual fue de 0,79 , representando también una correlación alta.

Ciertamente, el método de resolución data de muchos años, por lo que existen muchos trabajos que hacen referencia a este (Diago, Arnau y González, 2018; Esteven, Alonso y Salgado, 2016; Gasco, 2017); sin embargo, en la práctica muchos docentes suelen no tomar muy en cuenta la participación del estudiantado en la solución de los problemas, concentrándose más a desarrollar otros aspectos, y dejan que sus estudiantes sean quienes desarrollen los aspectos prácticos (resolución de problemas), creando desinterés y apatía hacia el aprendizaje de la asignatura; esto puede representar uno de los motivos por los cuales el estudiantado obtiene bajo rendimiento académico o desertan de los cursos durante los semestres regulares. La importancia del método de resolución de problemas en matemáticas radica en que a través de este se afianza el conocimiento, se desarrollan los procesos cognitivos, se garantiza el uso preciso del formalismo matemático, el proceso de formación se hace más a gusto y participativo y el alumnado adquiere mayor seguridad.

Se puede decir, que los resultados obtenidos en el presente trabajo son contrastados con investigaciones como las de Fonseca, Hernández y Mariño, (2017), la cual versa sobre el enfoque CPA (Concreto, Abstracto y Pictórico) en la resolución de problemas, indicando que, al aplicar una estrategia apropiada, se puede obtener mejoras en el rendimiento académico. También en la publicación de González (2000), se reportan mejoras al aplicar una metodología para la enseñanza por medio de la resolución de problemas, la cual constituye una herramienta conceptual. De igual forma, Leiva (2016) obtuvo resultados a favor del aprendizaje basado en problemas matemáticos, garantizando el desarrollo de las habilidades propias del pensamiento abstracto y de las competencias, tomando en cuenta únicamente la información que es de suma importancia para determinar las soluciones de los problemas. En ese sentido, lo que se persigue al aplicar métodos como el de resolución de problemas es incidir en el mejoramiento del rendimiento académico. 


\section{Conclusiones}

De acuerdo con análisis estadístico desarrollado, se determinó la influencia que ejerce el método de resolución de problemas sobre el desarrollo de actividades y el rendimiento académico sobre el grupo experimental, evidenciado en los resultados del pre-test y el post-test; la media de las calificaciones obtenidas en el pre-test favoreció al grupo control con una de diferencia de 0.39 por encima del grupo experimental; sin embargo, el estudiantado de ambos grupos presentó varias deficiencias al momento de desarrollar las soluciones. Después de aplicar el método de resolución de problemas al grupo experimental, la media de las calificaciones del post-test varió de 11.25 a 15.08 , por lo que fue notable el mejoramiento en el rendimiento académico del estudiantado de este grupo; mientras que la media obtenida por el grupo control fue de 11.42. El mejoramiento en el rendimiento académico del grupo experimental evidencia también el desarrollo de habilidades cognitivas en el estudiantado, lo cual fortalece el proceso de enseñanza y aprendizaje. Si el método de resolución de problemas es aplicado con flexibilidad, es decir, considerando las realidades del alumnado, sus capacidades y habilidades cognitivas, se logran resultados favorables concretados en el rendimiento académico, lo cual les permitirá enfrentar situaciones concretas a nivel social y profesional.

Además, se determinó que sí existe relación entre las habilidades y el rendimiento adquirido por el estudiantado universitario a través del método de resolución de problemas, con el coeficiente de correlación de Pearson obtenido de 0,771 .

\section{REFERENCIAS BIBLIOGRÁFICAS}

Calvo, M. M. (2008). Enseñanza eficaz de la resolución de problemas en matemáticas. Revista educación, 32(1), 123-138. Recuperado de https://www.redalyc.org/pdf/440/44032109.pdf

Chico, J., Gamboa, M., y Henríquez, L. (2017). El desarrollo de habilidades en la resolución de problemas trigonométricos de los estudiantes de segundo ciclo de la enseñanza secundaria angoleña. Revista Pertinencia Académica, (4), 49-68. Recuperado de: http://revista-academica.utb.edu.ec/index.php/pertacade/article/view/ 57

Carrasco, L., y Sánchez, M. (2016). Factores que favorecen la elección de las matemáticas como profesión entre mujeres estudiantes de la Universidad Veracruzana. Perfles Educativos, 38(151). 123-138. Recuperado de http://www. scielo.org.mx/pdf/peredu/v38n151/0185-2698-peredu-38-151-00123.pdf

Cerda, G., Pérez, C., Romera, E., Ortega, R., y Casas, J. (2017). Influencia de variables cognitivas y motivacionales en el rendimiento académico en matemáticas en estudiantes chilenos. Educación XXI. Revista de la Facultad de Educación, 20(2), 365-385. Recuperado de http://e-spacio.uned.es/fez/view/bibliuned:EducacionXXI-2017-2 $0-2-5075$

Diago, P., Arnau, D., y González, J. (2018). La resolución de problemas matemáticos en primeras edades escolares con Bee-bot. Matemáticas, educación y sociedad, 1(2), 36-50. Recuperado de http://mesjournal.es/ojs/index.php/m es/article/view/15

Echemendia, D., Ramos, I., y Delgado, N. (2017). La solución de problemas desde la enseñanza de la biología en carreras pedagógicas. Universidad y Sociedad, 9(5), 246-251. Recuperado de https://rus.ucf.edu.cu/index.php/rus/artic le/view/738

Esteven, J., Alonso, I., y Salgado, A. (2016). Resolución de problemas matemáticos en la licenciatura en educación matemática-física. REFCalE: Revista Electrónica Formación y Calidad Educativa, 4(1), 67-82. Recuperado de h ttp://refcale.uleam.edu.ec/index.php/refcale/article/view/481

Estrada, A. (2018). Estilos de aprendizaje y rendimiento académico. Revista Boletín Redipe, 7(7), 218-228. Recuperado de http://revista.redipe.org/index.php/1/article/view/536

Fonseca, R., Hernández, R., y Mariño, L. (2017). Enfoque CPA en la resolución de problemas para el aprendizaje de fracciones mediante el uso de software matemático. En R. Prada Núñez, P. Ramírez, C. Hernández, H. 
Gallardo, S. Mendoza y G. Rincón (Eds.). Encuentro Internacional en Educacio\#n Matemática (pp. 78-88). Cúcuta, Colombia: Universidad Francisco de Paula Santander. Recuperado de http://funes.uniandes.edu.co/1 $2773 /$

Gasco, J. (2017). La resolución de problemas aritmético-algebraicos y las estrategias de aprendizaje en matemáticas. Un estudio en educación secundaria obligatoria (ESO). RELIME. Revista latinoamericana de investigación en matemática educativa, 20(2), 167-192. doi: http://dx.doi.org/10.12802/relime.17.2022

González, T. (2000). Metodología para la enseñanza de las matemáticas a través de la resolución de problemas: un estudio evaluativo. Revista de Investigación educativa, 18(1), 175-199. Recuperado de https://revistas.um.es/ri e/article/view/121541

Kohler, J. (2013). Rendimiento académico, habilidades intelectuales y estrategias de aprendizaje en universitarios de Lima. Liberabit, 19(2), 277-288. Recuperado de: https://bit.ly/2FBE5yd

Leiva, F. (2016). ABP como estrategia para desarrollar el pensamiento lógico matemático en alumnos de educación secundaria. Sophia: Colección de Filosofía de la Educación, (21), 209-224. doi: http://dx.doi.org/10.17163/sop h.n21.2016.09

Martín, J., Martínez, P., Fernández, G., y Bravo, C. (2016). Analizando el desarrollo de las habilidades STEM a través de un proyecto ABP con arduino y su relación con el rendimiento académico. Repositorio digital Universidad Nacional Autónoma de México. Recuperado de https://bit.ly/3ccCt9X

Pifarré, M., y Sanuy, J. (2001). La enseñanza de estrategias de resolución de problemas matemáticos en la ESO: un ejemplo concreto. Enseñanza de las ciencias: Revista de investigación y experiencias didácticas, 19(2), 297-308. Recuperado de https://www.raco.cat/index.php/Ensenanza/article/download/21745/21579

Pino, E. (2013). La dimensión social de la universidad del siglo XXI creación del programa de aprendizaje-servicio en la Universidad Técnica de Ambato (Tesis doctoral). Universidad Complutense de Madrid, Madrid, España. Recuperado de https://eprints.ucm.es/22393/1/T34660.pdf

Roque, J. (2009). Influencia de la enseñanza de la matemática basada en la resolución de problemas en el mejoramiento del rendimiento académico. (Tesis de Maestría). Universidad Alas Peruanas, Lima, Perú. Recuperado de http:// cybertesis.unmsm.edu.pe/handle/20.500.12672/1704

Sabonete, J., Gamboa, M., y Mestre, U. (2016). Propuesta didáctica para el diseño de problemas matemáticos en escuelas angoleñas de segundo ciclo.Didasc@lia: Didáctica y Educación, 7(5), 155-164. https://dialnet.unirioj a.es/servlet/articulo?codigo $=6667087$

Sepúlveda, A., Medina, C., y Sepúlveda, D. (2009). La resolución de problemas y el uso de tareas en la enseñanza de las matemáticas. Educación matemática, 21(2), 79-115. Recuperado de http://www.scielo.org.mx/pdf/ed/v21n 2/v21n2a4.pdf

Serradó, A., Cardeñoso, J., y Azcárate, P. (2005). Los obstáculos en el aprendizaje del conocimiento probabilístico: su incidencia desde los libros de texto. Revista de investigación de educación estadística, 4(2), 59-81. Recuperado de https://n9.cl/h0rle

Villalobos, X. (2008). Resolución de problemas matemáticos: un cambio epistemológico con resultados metodológicos. REICE: Revista Iberoamericana sobre Calidad, Eficacia y Cambio en Educación, 6(3), 36-58. Recuperado de https://www.redalyc.org/pdf/551/55160303.pdf

\section{BY-NC-ND}

